\title{
Multi-Target Electrolyte Sensing Front-End for Wearable Physical Monitoring
}

\author{
Ivan Ny Hanitra, Francesca Criscuolo, Sandro Carrara and Giovanni De Micheli \\ Corresponding author: ivan.nyhanitra@epfl.ch \\ Integrated Systems Laboratory, EPFL, Switzerland
}

\begin{abstract}
Wearable physiology is an expanding field, especially for sports applications that steadily require an accurate monitoring of the physical status of athletes. This work presents the co-design and the realization of an hardware front-end that enables multi-sensing of up to four endogenous electrolytes. A pH and temperature readout circuit are included for sensor calibration. The platform is validated with potassium and sodium ions monitoring. The hardware is remotely controlled by an user interface that configures the sensor panel and collects the biological data through a Bluetooth link. The sizes of the frontend circuit allows its integration into a headband, suitable for wearable monitoring in sweat.

Index Terms-Wearable physiology, Multi-ion sensing, Potentiometric sensor readout, Temperature readout
\end{abstract}

\section{INTRODUCTION}

Since the last decade, wearable sensors are experiencing a fast growth with the spreading out of smart sensing systems aiming continuous monitoring of health and physical status of human beings [1]. Electrochemical sensors leverage the advancements in microtechnology to be miniaturized [2], and they enable non-invasive on-body sensing through biofluids such as sweat [3]. This is appealing for monitoring biological parameters of athletes who undergo important mineral loss during intense physical activity. Indeed, perspiration contains mostly minerals including sodium, potassium, calcium, magnesium and chloride. Depletion of sodium and potassium levels in sweat can lead to hypokalemia, hyponatremia, muscle cramps or dehydration [4], while calcium concentration indicates bone mineral loss [5]. In addition, chloride is a biomarker for cystic fibrosis [6]. As a result, a multi-target electrolyte sensing platform is needed for an optimal physiological balance. Ion sensing is typically performed by measuring the open circuit potential (OCP) between an electrode coated with an ion selective membrane (ISM) that selectively entraps the target analyte, and an inert reference electrode (RE) that has a stable potential. The measured cell potential is related to the activity of the tracked electrolyte through Nernst equation.

In [7], a multi-ion sensing platform is presented for wireless home-care systems. Yet, the size of the overall system is prohibitive for portable applications. A combined four-channels potentiometric/amperometric sensing platform is described in [8] where the hardware is mounted on a flexible polyamide substrate. However, recent work in [9] put in evidence the short

978-1-7281-3549-6/19/\$31.00 (C)2019 IEEE

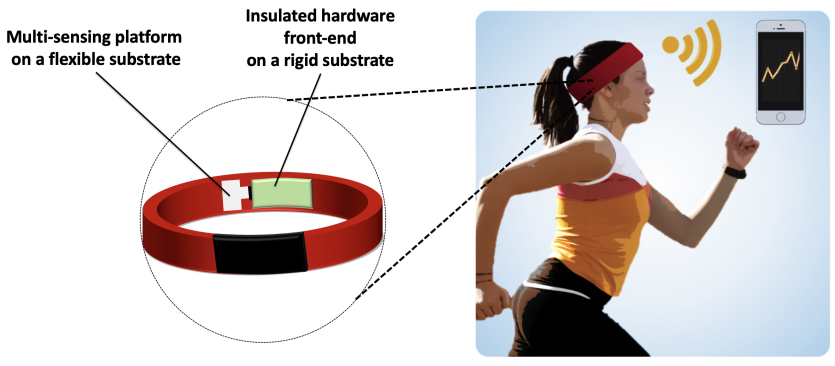

Fig. 1: Multi-target electrolyte sensing platform integrated in a smart headband and controlled remotely by an user interface.

lifespan of flexible printed circuit boards that are dynamically bent and folded. Advanced technologies and materials should be chosen carefully in order to have a longer wear and stable electrical connections on such type of substrate. The proposed solution is to build a small enough hardware platform that provides accurate on-body sensing performances.

In this work, a multi-target electrolyte sensing front-end is presented and characterized for wearable physical monitoring. The overall system is presented in Section II, while the front-end circuitry is described in Section III. It includes four ion-sensing channels plus $\mathrm{pH}$ and temperature readout circuitry. Then, the hardware is characterized with sodium and potassium sensor calibrations in Section IV. Conclusions are reported in Section V.

\section{SYSTEM OVERVIEW}

Wearable physiology in sweat is performed on the forehead that is easily accessible with a headband. It is a relatively flat zone, relaxing the constraints of having a fully mechanicallyflexible sensing platform. The overall sensing platform is presented in Fig. 1. It consists of the multi-target electrolyte sensor panel and the hardware front-end that acquires, processes and transmits the physiological information to a control and display terminal via a wireless link. The different system blocks are described hereunder by emphasizing a co-design of the readout front-end for a seamless sensor integration.

\section{A. Sensing platform}

The multi-sensor panel is manufactured on a polyamide substrate. It comprises four gold ion sensing electrodes (ISEs) that are patterned with standard lithographic technology. A 


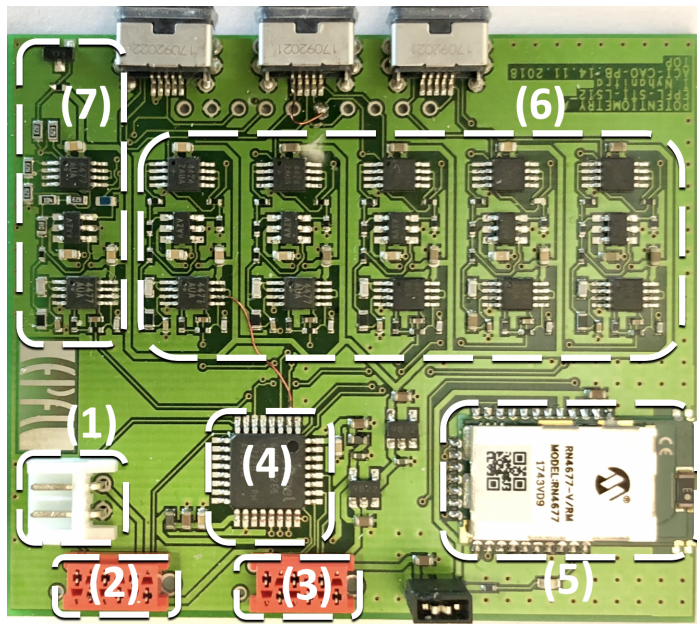

Fig. 2: Different blocks of the potentiometric board: (1) socket for the power supply, (2) Programming/Debugging Interface, (3) UART serial port and alternative power supply, (4) microcontroller unit, (5) Bluetooth module, (6) four-channels potentiometric readout and $\mathrm{pH}$ readout circuitry, (7) temperature readout block.

$\mathrm{RE}$ is fabricated by chloridation of a silver electrode. It is shared between the four sensors. Furthermore, $\mathrm{pH}$ and temperature are two important parameters for sensor calibration and controlling the stability of the ISM [10]. A pH sensor is implemented with a platinum electrode coated with an iridium oxide film. The OCP of the metal oxide film varies linearly with the in-situ $\mathrm{pH}$ [11]. Local temperature is monitored with a resistive thermal device (RTD). Namely, a conductive material which resistance varies predictably with temperature [12]. The nominal resistance of the device depends on the material chosen, its thickness, width and overall length. With a gold serpentine-shaped structure, the RTD has a resistance of $600 \Omega$ at $20^{\circ} \mathrm{C}$.

\section{B. Hardware front-end}

The hardware front-end is manufactured on a $1.6 \mathrm{~mm}$ FR4 substrate with $54 \times 64 \mathrm{~mm}$ size. It could be further shrunk by more aggressive placement and routing, but the size of the batteries $(62 \times 44 \mathrm{~mm})$ is the restricting dimension in system integration. In spite of that, the hardware and its supply fit into commercial headbands. The different blocks constituting the readout front-end are listed and highlighted in Fig. 2. The sensor panel is interfaced with the hardware through a ten-positions flat-flex type edge connector. Yet, in Fig. 2, micro USB-B connectors are soldered for prototyping. Then, a low-power ATxmega32E5 microcontroller unit (MCU) is the central processing unit. It embeds a 12-bit resolution analogto-digital converter (ADC) able to convert up to $300 \mathrm{ksps}$. It is calibrated beforehand. The serial port of the MCU is used to relay the signals to a RN4677 Bluetooth Low Energy (BLE) module that is connected to a remote interface. In the following, Matlab ${ }^{\circledR}$ serial port of a Personal Computer is used for both setting up the sensing channels and collecting

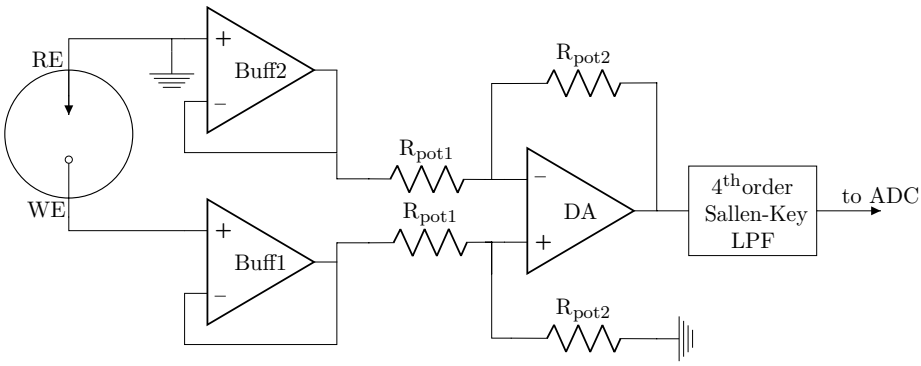

Fig. 3: Readout circuit for potentiometric sensing.

the measured data. A UART protocol at 115'200 baud rate, with start and stop tags is implemented to ensure safe data transmission. The board is powered with a lithium-ion battery of $3.7 \mathrm{~V}$ nominal voltage and $2000 \mathrm{mAh}$ capacity. The power consumption of the system is of $175 \mathrm{~mW}$ in normal sensing mode.

\section{FRONT-END CIRCUIT ARCHITECTURE}

The analog front-end circuit implemented for potentiometric sensing and temperature monitoring is presented in this section. Off-the-shelf components are used for the amplifiers, voltage reference and passive elements.

\section{A. Potentiometric readout}

The readout circuitry of one potentiometric sensing channel is displayed in Fig. 3. Five blocks are replicated: four are measuring the floating potential of four ISEs against a grounded $\mathrm{RE}$, while one channel is dedicated to $\mathrm{pH}$ monitoring.

Open circuit measurements necessitate voltage buffers with high input impedance in order to isolate the sensor from the electronic circuit. The used buffers draw $500 \mathrm{pA}$ input bias current. This non-null polarization current is required to improve the lower limit of detection (LOD), to increase sensitivity and to reduce potential drift of the sensor [13]. Next, an amplification stage of gain 3.9 is implemented with a single-ended differential amplifier. The full ADC input range is used to resolve the signal that spans in $[0 ; 500] \mathrm{mV}$ range. The signal conditioning path includes a low-pass filter as well. A $4^{\text {th }}$ order Sallen-Key filter topology is chosen for anti-aliasing purposes and to attenuate high-frequency noise. The design of the filter is presented in [9], where the unity-gain active filter features a $-3 \mathrm{~dB}$ cut-off at $200 \mathrm{~Hz}$. It is a good trade-off considering the slow biosignals $(1 \mathrm{to} 100 \mathrm{~Hz})$ and component sizing.

\section{B. Temperature readout}

The in-situ temperature is monitored with a RTD device whose resistance varies predictably with temperature [12]. The range of interest for the RTD values is from 200 to $1600 \Omega$. A typical way to measure the resistance of the RTD is to drive the device with a current source and to measure the voltage drop across it. In [8], a simple voltage divider is implemented, but accuracy is degraded by loading effects. Moreover, if the magnitude of the current source is too high, the RTD element 


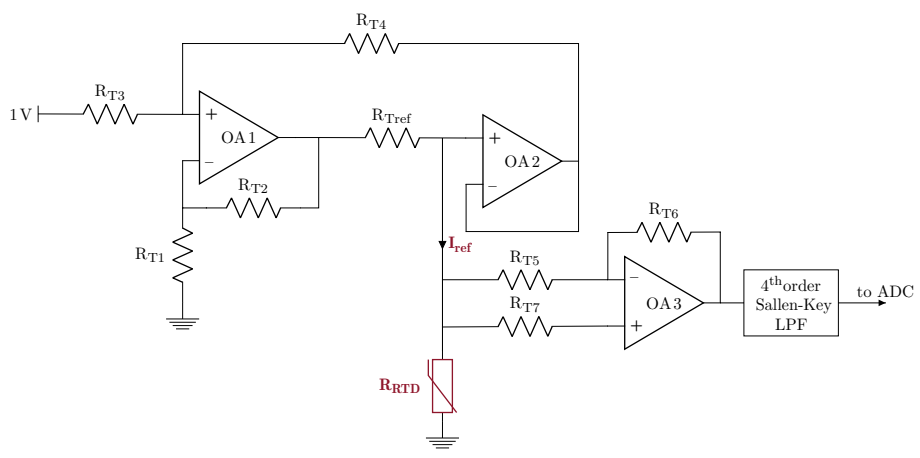

Fig. 4: RTD readout circuit.

will dissipate power and start to self-heat. Consequently, care should be taken for the design of the current generator. Howland current pumps are voltage-controlled current sources that are commonly used in electrical stimulation systems or electrical impedance tomography [14]. A modified version of the improved Howland current source [15] is presented in Fig. 4. A precision voltage reference of $1 \mathrm{~V}$ is the controlling input. Operational amplifier $O A l$ senses the input voltage and the feedback signal differentially, and sets a voltage drop of $1 \mathrm{~V}$ across $\mathrm{R}_{\text {Tref }}=1 \mathrm{k} \Omega$. The current source is sized so as to reduce the effects of mismatch between resistors and tolerance of $R_{\text {Tref }}$ value. Thus, a floating current source $I_{\text {ref }}=1 \mathrm{~mA}$ is generated, independently of its load, in the load resistor range of interest. A stable current reference is needed for highly linear R-to- $\mathrm{V}$ conversion, therefore $O A 2$ is used to increase the output impedance of the current source. The output impedance is computed as $Z_{\text {out }}=\frac{R_{\text {Tref }}}{1-\alpha}$, with

$$
\alpha=\mathrm{A}_{1} \cdot \frac{\left(\mathrm{R}_{\mathrm{T} 1}+\mathrm{R}_{\mathrm{T} 2}\right) \cdot \mathrm{R}_{\mathrm{T} 3}}{\left(\mathrm{R}_{\mathrm{T} 3}+\mathrm{R}_{\mathrm{T} 4}\right) \cdot\left(\mathrm{R}_{\mathrm{T} 1}+\mathrm{R}_{\mathrm{T} 2}+\mathrm{A}_{1} \mathrm{R}_{\mathrm{T} 1}\right)} \cdot \frac{\mathrm{A}_{2}}{1+\mathrm{A}_{2}}
$$

where $\mathrm{A}_{1}$ and $\mathrm{A}_{2}$ are the open loop gain of $O A 1$ and $O A 2$, respectively. An output impedance of $333 \mathrm{M} \Omega$ is estimated with the proposed design. $O A 3$ is implemented for high precision measurement. It cancels the contributions of wire and contact resistances of the RTD that could be non-negligible considering the small values of $\mathrm{R}_{\mathrm{RTD}}$. It can be easily shown that the balanced opamp ensures that output voltage represents the voltage drop across the RTD. A $4^{\text {th }}$ order Sallen-Key filter similar to the one for potentiometric readout is included prior to the sampler.

The R-to-V converter is characterized by sampling the filtered output voltage with different high-precision value load resistor $\mathrm{R}_{\mathrm{RTD}}$, in the range $[50 ; 2000] \Omega$. The calibration of the converter is reported in Fig. 5, exhibiting a sensitivity of $1.83 \mathrm{mV} / \Omega \pm 4 \mu \mathrm{V} / \Omega$ that covers the full ADC input range.

\section{Electrochemical CHARACTERIZATION}

In this section, the hardware front-end is characterized with potassium and sodium sensors. The measurements are done in-vitro, in water samples.

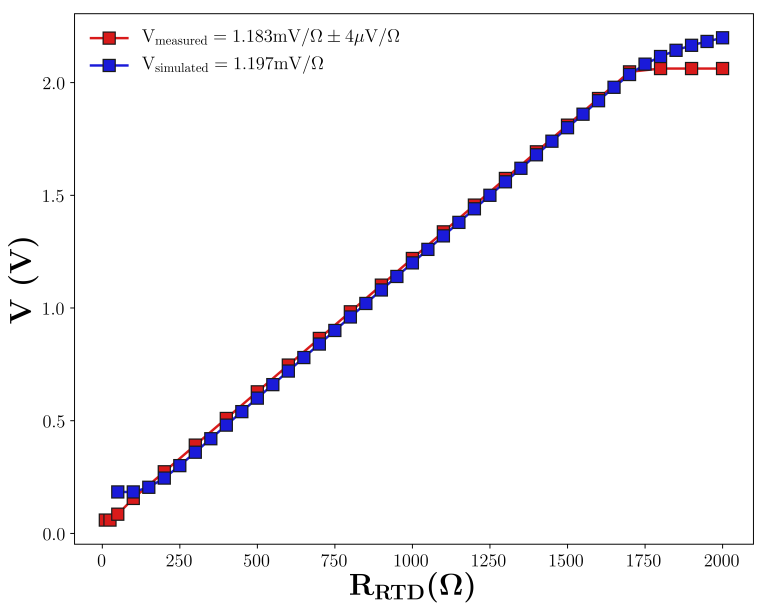

Fig. 5: Calibration of the temperature readout circuitry.

\section{A. Materials and methods}

Electrode functionalization for sodium and potassium ions measurements is described in the following. The support for the ISEs are circular working electrode $(4 \mathrm{~mm} \varnothing$ in platinum) of commercial Screen-Printed Electrodes (SPEs) from Metrohm.

The potassium-selective membrane was prepared by dissolving $100 \mathrm{mg}$ of mixture consisting of $33.00 \mathrm{wt} \%$ Poly(vinyl chloride) high molecular weight, $1.00 \mathrm{wt} \%$ potassium ionophore I, $0.50 \mathrm{wt} \%$, Potassium tetrakis(4chlorophenyl)borate and $65.50 \mathrm{wt} \%$ bis(2-ethylhexyl)sebacate (DOS) in $1 \mathrm{~mL}$ of Tethraydrofuran (THF). The sodiumselective membrane was instead prepared by dissolving $100 \mathrm{mg}$ of mixture consisting of $33.00 \mathrm{wt} \%$ Poly(vinyl chloride) high molecular weight, $0.70 \mathrm{wt} \%$ sodium ionophore $\mathrm{X}, 0.20 \mathrm{wt} \%$, Potassium tetrakis(4-chlorophenyl)borate and $66.10 \mathrm{wt} \%$ 2-Nitrophenyl octyl ether in $1 \mathrm{~mL}$ of Tethraydrofuran (THF).

A $10 \mu \mathrm{L}$ drop of aforementioned solutions was then dropcasted onto respective SPE, after electrochemical nanostructuration of the surface, as described in [16]. The electrodes were left to dry overnight in dark conditions. A conditioning procedure in $0.01 \mathrm{M} \mathrm{KCl}$ and $0.01 \mathrm{M} \mathrm{NaCl}$ for $24 \mathrm{~h}$ was performed before each measurement.

In all the presented measurements, an $\mathrm{Ag} / \mathrm{AgCl}$ doublejunction electrode from Methrom is used as reference electrode. Results are compared with an Autolab (Metrohm) potentiostat.

\section{B. Potassium sensor calibration}

The calibration of the potassium sensor with the hardware front-end is reported in Fig. 6. $\mathrm{KCl}$ is gradually injected every $50 \mathrm{~s}$, from $10^{-9} \mathrm{M}$ to $0.1 \mathrm{M}$. The time trace of the acquired OCP is displayed in the upper left inset of Fig. 6. A nearNernstian calibration curve is obtained with a sensitivity of $57.3 \pm 1.1 \mathrm{mV} /$ decade. The lower LOD is computed according 


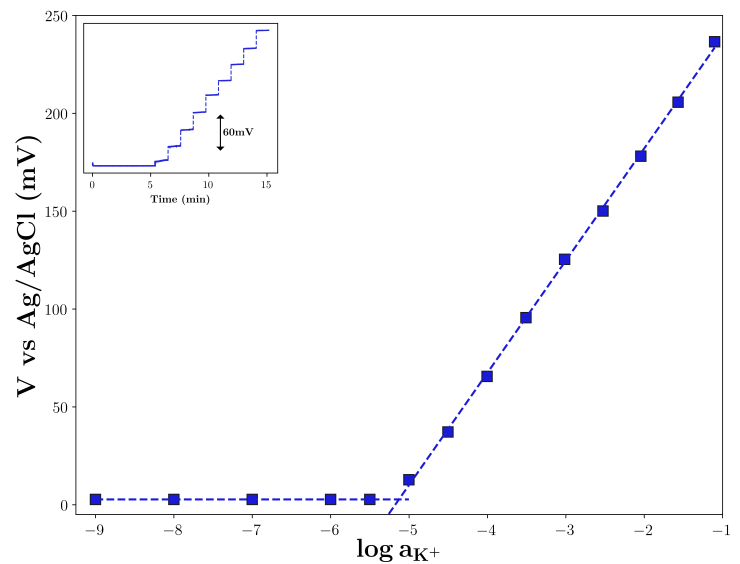

Fig. 6: Potassium sensor calibration in water background electrolyte.

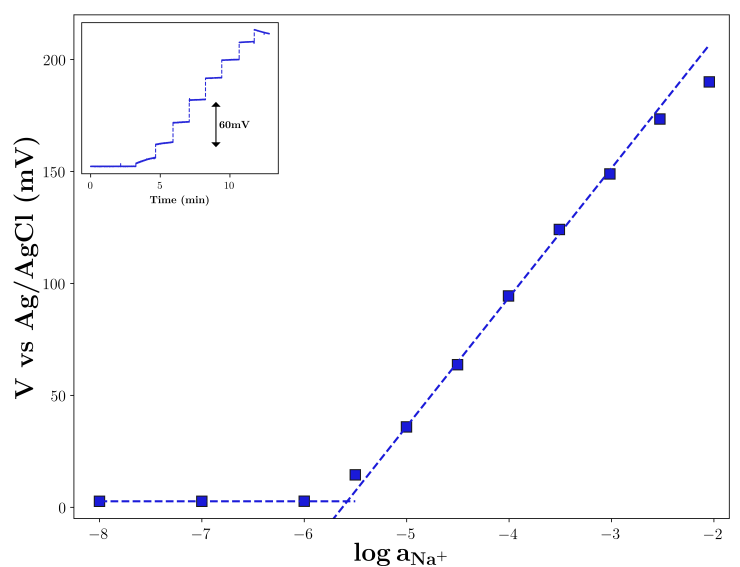

Fig. 7: Sodium sensor calibration in water background electrolyte.

to IUPAC definition, as the intersection of the extrapolated linear portions of the calibration curve. The resulting lower LOD is of $5 \pm 1 \mu \mathrm{M}$. This range is satisfactory considering the physiological relevant concentrations of potassium in sweat in $1-40 \mathrm{mM}$. The same potassium sensor calibration is performed with an Autolab potentiostat, providing sensitivity of $57.8 \pm 1.2 \mathrm{mV} /$ decade and lower LOD of $4.2 \pm 1 \mu \mathrm{M}$.

\section{Sodium sensor calibration}

Likewise, the calibration of the sodium sensor with the hardware front-end is reported in Fig. 7. The OCP acquisition exhibits a sub-Nernstian behavior with a sensitivity of $57.7 \pm 1.5 \mathrm{mV} /$ decade and lower LOD of $2.5 \pm 1 \mu \mathrm{M}$. The relevant physiological range of sodium in sweat being of $1-160 \mathrm{mM}$. Measurements with an Autolab potentiostat yield sensitivity of $58.5 \pm 1 \mathrm{mV} /$ decade and lower LOD of $3.5 \pm 1 \mu \mathrm{M}$.

\section{CONCLUSION}

The co-design and realization of a front-end for multiions sensing is presented. The hardware is characterized with potassium and sodium sensors, providing performances similar to those of a bulky commercial potentiostat. The frontend includes a temperature sensor that is crucial for sensor calibration. Its electrical characterization yields almost perfect linearity and optimal sensitivity, in the resistance range of the envisioned RTD. The front-end is planned to be interfaced to a multi-ion sensing platform on a flexible substrate, that is currently under manufacturing process at EPFL clean-room facilities. The overall integrated system will pave the way to wearable physiology applications in sweat.

\section{ACKNOWLEDGEMENT}

This research is supported by H2020 ERC 2014 ADG 669354 CyberCare.

\section{REFERENCES}

[1] S. C. Mukhopadhyay, "Wearable sensors for human activity monitoring: A review," IEEE Sensors Journal, vol. 15, no. 3, pp. 1321-1330, March 2015.

[2] N. J. Ronkainen, H. B. Halsall, and W. R. Heineman, "Electrochemical biosensors," Chemical Society Reviews, vol. 39, no. 5, p. 1747, 2010.

[3] J. Kim, A. S. Campbell, B. E.-F. de Ávila, and J. Wang, "Wearable biosensors for healthcare monitoring," Nature Biotechnology, 2019.

[4] D. B. Speedy, T. D. Noakes, and C. Schneider, "Exercise-associated hyponatremia: A review," Emergency Medicine, vol. 13, no. 1, pp. 17$27,2001$.

[5] K. RC, W. KD, S. ML, and et al, "Changes in bone mineral content in male athletes: Mechanisms of action and intervention effects," JAMA, vol. 276, no. 3, pp. 226-230, 1996.

[6] J. V. Pagaduan, M. Ali, M. Dowlin, L. Suo, T. Ward, F. Ruiz, and S. Devaraj, "Revisiting sweat chloride test results based on recent guidelines for diagnosis of cystic fibrosis," Practical Laboratory Medicine, vol. 10, pp. $34-37,2018$.

[7] J. F. Cheng, J. C. Chou, T. P. Sun, S. K. Hsiung, and H. L. Kao, "Study on a multi-ions sensing system for monitoring of blood electrolytes with wireless home-care system," IEEE Sensors Journal, vol. 12, no. 5, pp. 967-977, 2012.

[8] W. Gao, S. Emaminejad, H. Y. Y. Nyein, S. Challa, K. Chen, A. Peck, H. M. Fahad, H. Ota, H. Shiraki, D. Kiriya, D.-H. Lien, G. A. Brooks, R. W. Davis, and A. Javey, "Fully integrated wearable sensor arrays for multiplexed in situ perspiration analysis," Nature, vol. 529, no. 7587, pp. 509-514, 2016.

[9] I. Ny Hanitra, L. Lobello, F. Stradolini, A. Tuoheti, F. Criscuolo, T. Kilic, D. Demarchi, S. Carrara, and G. De Micheli, "A Flexible Front-End for Wearable Electrochemical Sensing," in IEEE International Symposium on Medical Measurements and Applications, Proceedings, 2018.

[10] F. Coldur and M. Andac, "All-Solid-State Polyvinyl Chloride Membrane Lithium-Selective Electrode with Improved Selectivity and Its Application in Serum Lithium Assay," Sensor Letters, vol. 9, no. 5, pp. 17381744, 2012

[11] W. Olthuis, M. Robben, P. Bergveld, M. Bos, and W. van der Linden, "ph sensor properties of electrochemically grown iridium oxide," Sensors and Actuators B: Chemical, vol. 2, no. 4, pp. 247 - 256, 1990.

[12] M. S. V. Dusen, "Platinum-Resistance Thermometry At Low Temperatures," Journal of the American Chemical Society, vol. 47, no. 2, pp. 326-332, 1925.

[13] E. Lindner and R. E. Gyurcsányi, "Quality control criteria for solidcontact, solvent polymeric membrane ion-selective electrodes," Journal of Solid State Electrochemistry, vol. 13, no. 1, pp. 51-68, 2009.

[14] K. Sooksood, T. Stieglitz, and M. Ortmanns, "An active approach for charge balancing in functional electrical stimulation," IEEE Transactions on Biomedical Circuits and Systems, vol. 4, no. 3, pp. 162-170, June 2010.

[15] K. F. Morcelles, V. G. Sirtoli, P. Bertemes-Filho, and V. C. Vincence, "Howland current source for high impedance load applications," Review of Scientific Instruments, vol. 88, no. 11, 2017.

[16] F. Criscuolo, I. Taurino, F. Stradolini, S. Carrara, and G. D. Micheli, "Highly-stable li+ ion-selective electrodes based on noble metal nanostructured layers as solid-contacts," Analytica Chimica Acta, vol. 1027, pp. $22-32,2018$. 